\title{
Formation of Long-Lived Color Centers for Broadband Visible Light Emission in Low-Dimensional Layered Perovskites
}

\author{
Edward P. Booker, ${ }^{*}{ }^{\dagger}$ Tudor H. Thomas, ${ }^{\dagger}$ Claudio Quarti, ${ }^{\ddagger 0}$ Michael R. Stanton, ${ }^{\dagger}$ \\ Cameron D. Dashwood, ${ }^{\dagger}$ Alexander J. Gillett, ${ }^{\dagger}$ Johannes M. Richter, ${ }^{\dagger}$ Andrew J. Pearson, ${ }^{\dagger}$ \\ Nathaniel J. L. K. Davis, ${ }^{\dagger}$ Henning Sirringhaus, ${ }^{\dagger}$ Michael B. Price, ${ }^{\dagger}$ Neil C. Greenham, ${ }^{\dagger}$ (๑) David Beljonne, ${ }^{\dagger}$ \\ Siân E. Dutton, ${ }^{\dagger}$ and Felix Deschler* ${ }^{\dagger}$ \\ ${ }^{\dagger}$ Cavendish Laboratory, University of Cambridge, CB3 0HE Cambridge, United Kingdom \\ ${ }^{\ddagger}$ Laboratory for Chemistry of Novel Materials, University of Mons, Place du Parc 20, B-7000 Mons, Belgium
}

Supporting Information

\begin{abstract}
We investigate the origin of the broadband visible emission in layered hybrid lead-halide perovskites and its connection with structural and photophysical properties. We study $\langle 001\rangle$ oriented thin films of hexylammonium (HA) lead iodide, $\left(\mathrm{C}_{6} \mathrm{H}_{16} \mathrm{~N}\right)_{2} \mathrm{PbI}_{4}$, and dodecylammonium (DA) lead iodide, $\left(\mathrm{C}_{12} \mathrm{H}_{28} \mathrm{~N}\right)_{2} \mathrm{PbI}_{4}$, by combining first-principles simulations with time-resolved photoluminescence, steady-state absorption and X-ray diffraction measurements on cooling from 300 to $4 \mathrm{~K}$. Ultrafast transient absorption and photoluminescence measurements are used to track the formation and recombination of emissive states. In addition to the excitonic photoluminescence near the absorption edge, we find a red-shifted, broadband (full-width at half-maximum of about $0.4 \mathrm{eV}$ ), emission band below $200 \mathrm{~K}$, similar to emission from $\langle 110\rangle$ oriented bromide $2 \mathrm{D}$ perovskites at room temperature. The lifetime of this subband-gap emission exceeds that of the excitonic transition by orders of

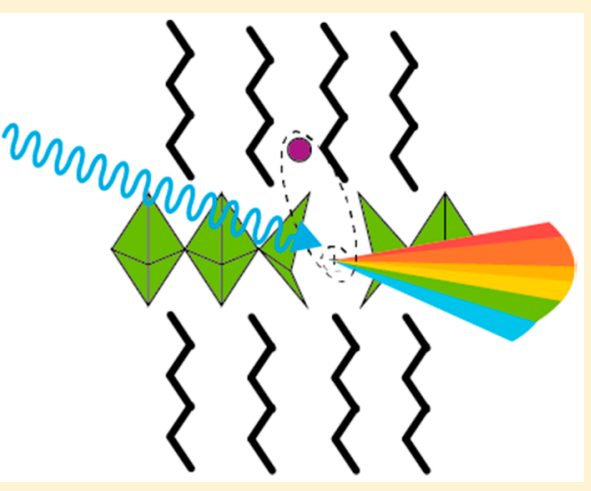
magnitude. We use X-ray diffraction measurements to study the changes in crystal lattice with temperature. We report changes in the octahedral tilt and lattice spacing in both materials, together with a phase change around $200 \mathrm{~K}$ in $\mathrm{DA}_{2} \mathrm{PbI}_{4}$. DFT simulations of the $\mathrm{HA}_{2} \mathrm{PbI}_{4}$ crystal structure indicate that the low-energy emission is due to interstitial iodide and related Frenkel defects. Our results demonstrate that white-light emission is not limited to $\langle 110\rangle$ oriented bromide 2D perovskites but a general property of this class of system, and highlight the importance of defect control for the formation of low-energy emissive sites, which can provide a pathway to design tailored white-light emitters.
\end{abstract}

\section{INTRODUCTION}

Lead halide perovskite solar cell and light-emitting diode (LED) efficiencies have increased substantially in recent years, ${ }^{1-3}$ but their instability in air $^{4}$ and ultraviolet light ${ }^{5}$ challenges commercial application. Whole or partial substitution of the standard cation amines in the 3D bulk perovskites ${ }^{6,7}$ with longer aliphatic chains was suggested to alleviate the instability. Use of such larger-cation amines changes the crystal structure into a $2 \mathrm{D}$ perovskite ${ }^{8}$ with semiconducting lead-halide perovskite sheets separated by a layer of insulating aliphatic chain (organic spacer). Recently, examples of $2 \mathrm{D}$ perovskite $\mathrm{LEDs}^{8}$ and $2 \mathrm{D} / 3 \mathrm{D}$ mixed perovskite solar cells have been reported, but the solar cell efficiencies still fall behind their pure $3 \mathrm{D}$ analogues, even 2D/3D mixtures, which allow for separation of initial excitonic states. 9,10

In recent years, there have been several accounts of whitelight emission from $\langle 110\rangle$ oriented $2 \mathrm{D}$ perovskites ${ }^{11-17}$ and one example from $\langle 100\rangle$ oriented $2 \mathrm{D}$ perovskites. ${ }^{18}$ White-light emission is the formation of a red-shifted broad emission peak below the band gap. White light emission from phosphors is an attractive feature in LEDs as it allows for application in domestic lighting. However, the detailed mechanism of whitelight emission has a complex origin. In particular, white-lightemitting hybrid perovskites contain widely differing organic spacers and show large differences in crystal structure. This has so far limited controlled studies into the white-light emission in hybrid perovskites, in particular with regard to compositional and structural effects.

Here we report on white-light emission in two $\langle 001\rangle$ oriented 2D-perovskite materials, hexylammonium lead iodide $\left(\mathrm{HA}_{2} \mathrm{PbI}_{4}\right)$ and dodecylammonium lead iodide $\left(\mathrm{DA}_{2} \mathrm{PbI}_{4}\right)$. We study the steady-state and transient optical behavior of these materials from room temperature down to $12 \mathrm{~K}$ and find that below $200 \mathrm{~K}$ charge recombination results in white-light emission. We use temperature as a parameter to explore the origin of the white-light emission. Steady-state optical absorbance and XRD measurements, in the range from 300 to $12 \mathrm{~K}$, demonstrate that the low-energy emission does not originate from a new bulk phase. Ultrafast photoluminescence

Received: September 25, 2017

Published: November 20, 2017 
and ultrafast transient absorption measurements confirm the excitonic origin of the emission and demonstrate that the subband-gap state is not transiently populated at room temperature. First-principles calculations indicate that the broad, longlived emission seen at low temperatures in is due to the lightinduced formation of localized trap states, associated with interstitial iodide and iodide Frenkel defects that act as color centers in the crystal. Our results provide a rational approach to develop new perovskite compounds with tunable color centers for efficient white-light emitting LED devices.

\section{RESULTS}

$\mathrm{HA}_{2} \mathrm{PbI}_{4}$ and $\mathrm{DA}_{2} \mathrm{PbI}_{4}$ thin films were synthesized from solutions of alkylammonium iodide salts and lead iodide. The room-temperature structures of both $\mathrm{HA}_{2} \mathrm{PbI}_{4}{ }^{19}$ and $\mathrm{DA}_{2} \mathrm{PbI}_{4}{ }^{20}$ have previously been reported from single-crystal measurements. We find good agreement with the reported diffraction peaks and our room temperature XRD measurements on thin films. In both cases, the structure consists of sheets of cornersharing $\mathrm{PbI}_{4}{ }^{2-}$ octahedral (Figure 1a), which are separated by
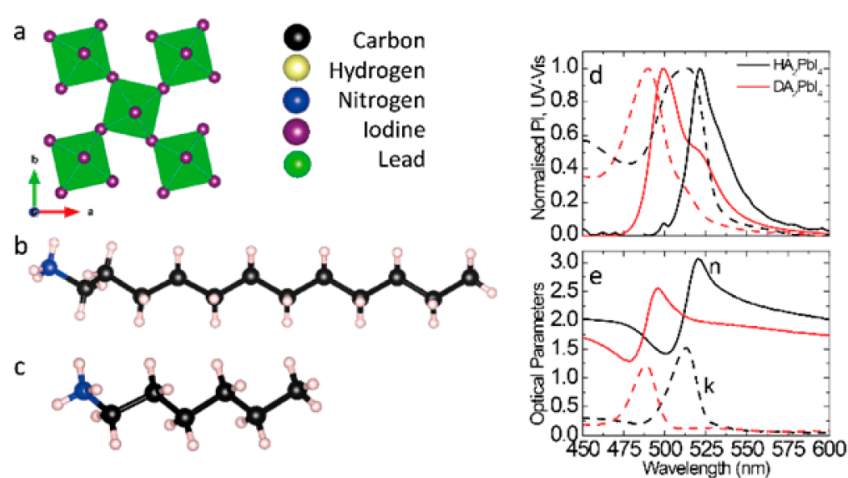

Figure 1. Chemical structure and optical properties of layered hybrid lead-halide perovskites. (a) Polyhedral model showing the connectivity of the $\mathrm{PbI}_{4}{ }^{2-}$ sheets in $\mathrm{HA}_{2} \mathrm{PbI}_{4}$ and $\mathrm{DA}_{2} \mathrm{PbI}_{4} \cdot(\mathrm{b}, \mathrm{c})$ Ball and stick models showing the chemical structures of dodecylammonium and hexylammonium, respectively. (d) Steady-state optical absorption (dashed line) and photoluminescence (solid line) of the 2D perovskites at room temperature. (e) Steady-state real ( $n$, solid line) and imaginary $(k$, dashed line) parts of the refractive index of the $2 \mathrm{D}$ perovskites.

alkylammonium spacers (Figure $1 \mathrm{~b}, \mathrm{c}$ ). We find substantial preferential alignment with lead iodide layers parallel to the glass substrate (Supporting Information Figures 1, 2, and 9). To enable quantitative analysis of the crystal structure, powder samples were prepared from drop-cast films. Rietveld analysis ${ }^{21}$ was carried out using the TOPAS program ${ }^{22}$ on the roomtemperature data demonstrating that, at room temperature, $\mathrm{HA}_{2} \mathrm{PbI}_{4}$ powder belongs to the monoclinic $P 2_{1} / a$ space group $^{19}$ with lattice parameters of $a=8.693(5) \AA, b=8.911(5)$ $\AA, c=16.338(10) \AA$, and $\beta=94.85(3)^{\circ}$ which corresponds to low-temperature film measurements by Billing and Lemmerer. ${ }^{19} \mathrm{DA}_{2} \mathrm{PbI}_{4}$ belongs to the orthorhombic $\mathrm{Pbca}$ space group ${ }^{20}$ with lattice parameters $a=8.843(2) \AA, b=8.5023(18) \AA, c=$ 48.988(9) Å. Tables summarizing the Rietveld analysis are included in the Supporting Information (Tables 4 and 5), as well as representative XRD spectra and the respective Rietveld fits (Supporting Information Figure S14).

Steady-state optical properties of the thin films are shown in Figure 1d,e. The primary steady-state photoluminescence peak is at $525 \mathrm{~nm}$ for $\mathrm{HA}_{2} \mathrm{PbI}_{4}$, and $500 \mathrm{~nm}$ for $\mathrm{DA}_{2} \mathrm{PbI}_{4}$, however both show a slight broadening in the emission below the main narrow peak. The photoluminescence quantum efficiency (PLQE) at room temperature was measured in an integrating sphere to be $3.2 \%$ for $\mathrm{DA}_{2} \mathrm{PbI}_{4}$ and $0.37 \%$ for $\mathrm{HA}_{2} \mathrm{PbI}_{4}$. The optical constants were measured with ellipsometry (Figure 1e) and agree with previous reports. ${ }^{23,24}$ Both show one dominant oscillator near the band edge, in agreement with a strongly excitonic, phase-pure system.

We used temperature to tune the structural and optical properties of the materials. Steady-state and time-resolved (TCSPC) photoluminescence measurements were taken at temperatures between 300 to $4 \mathrm{~K}$ (Figure 2). From room temperature
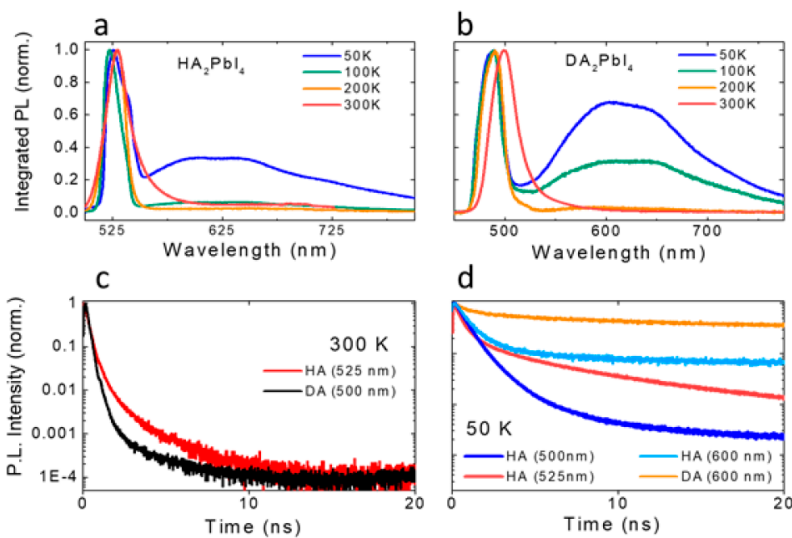

d

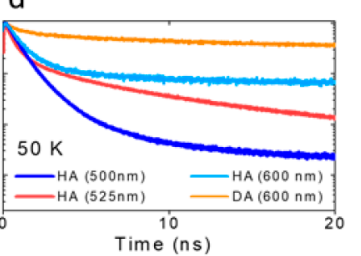

Figure 2. Temperature-dependent transient $\mathrm{PL}$ of (a) $\mathrm{HA}_{2} \mathrm{PbI}_{4}$ and (b) $\mathrm{DA}_{2} \mathrm{PbI}_{4}$ thin films from TC-SPC measurements. The spectra show a dominant PL peak at room temperature, with the appearance of a low-energy PL feature with a broad peak between 600 and $650 \mathrm{~nm}$ at lower temperatures. PL kinetics at (c) $300 \mathrm{~K}$ and (d) $50 \mathrm{~K}$ for selected emission wavelengths.

down to $200 \mathrm{~K}$ the PL spectra are dominated by emission close to the optical band edge of the materials, which we attribute to the recombination of photoexcited excitonic states. Below 200 $\mathrm{K}$ we find the appearance of a broad feature that is red-shifted from the room-temperature $\mathrm{PL}$ by $0.2 \mathrm{eV}$ for $\mathrm{HA}_{2} \mathrm{PbI}_{4}$, and 0.4 $\mathrm{eV}$ for $\mathrm{DA}_{2} \mathrm{PbI}_{4}$. The feature has a fwhm of around $0.4 \mathrm{eV}$ and is centered at roughly $625 \mathrm{~nm}$ for both materials $(\sim 0.7 \mathrm{eV}$ below the electronic band gap of $\mathrm{HA}_{2} \mathrm{PbI}_{4}$ as estimated by Tanaka et al. ${ }^{25}$ ). We take this as a first indication that the lowenergy emission shares a common origin in both materials, despite the differences in crystal structure and spacing between the inorganic perovskite layers. The time-resolved PL decays (Figure 2c,d) show that the red-shifted feature exhibits a significantly longer lifetime than the excitonic recombination near the band edge, with residual emission present after several hundreds of nanoseconds (Supporting Information Figure 4). The formation of the low-energy emission cannot be resolved within the resolution (120 ps) of our TC-SPC measurements, which sets an upper bound for the transfer time of the initially excited states near the band edge to the red-shifted, lowerenergy state. Lifetimes of the room temperature PL are $0.25 \mathrm{~ns}$ in $\mathrm{HA}_{2} \mathrm{PbI}_{4}$ and $0.47 \mathrm{~ns}$ in $\mathrm{DA}_{2} \mathrm{PbI}_{4}$. At $50 \mathrm{~K}$ the main $\mathrm{PL}$ peak decays with a time constant of $0.75 \mathrm{~ns}$ in $\mathrm{HA}_{2} \mathrm{PbI}_{4}$ and $0.5 \mathrm{~ns}$ in $\mathrm{DA}_{2} \mathrm{PbI}_{4}$ Supporting Information Figure 4). The lifetimes of the low-energy emission increase for both materials for lower temperatures however the lifetime exceeded the range of the measurement so cannot be accurately determined (Supporting Information Figures 5 and 6). 
The emission intensity from the low-energy feature is temperature dependent (Figure 2a,b) and increases by 3 orders of magnitude from 200 to $50 \mathrm{~K}$. The temperature dependence of the integrated emission is shown in Supporting Information Figure 3. By comparing the PL spectra at $50 \mathrm{~K}$ obtained from TC-SPC to room-temperature TC-SPC and PLQE measurements we extrapolated the PLQE at $50 \mathrm{~K}$ to be $6.1 \%$ in $\mathrm{DA}_{2} \mathrm{PbI}_{4}$ and $2.9 \%$ in $\mathrm{HA}_{2} \mathrm{PbI}_{4}$ at $50 \mathrm{~K}$. In $\mathrm{DA}_{2} \mathrm{PbI}_{4}, 32 \%$ of the emission came from low-energy emission and in $\mathrm{HA}_{2} \mathrm{PbI}_{4}$ this proportion was $15 \%$

To further investigate the origin of the red-shifted emission feature and a possible structural cause we collected optical absorption spectra (Figure 3) and powder XRD spectra (Figure
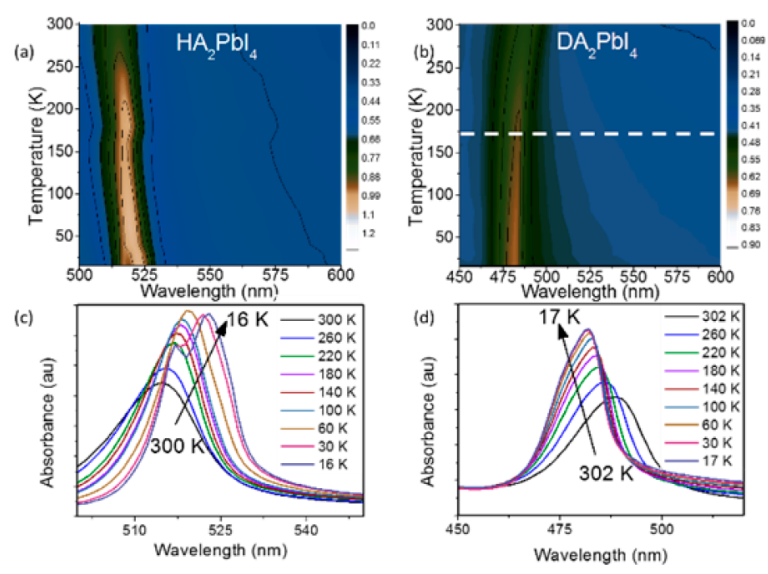

Figure 3. (a,b) Temperature-dependent optical absorption spectrum under cooling from 300 to $12 \mathrm{~K}$ for (a) $\mathrm{HA}_{2} \mathrm{PbI}_{4}$ and (b) $\mathrm{DA}_{2} \mathrm{PbI}_{4}$. (c,d) Temperature-dependent changes in the absorption peak region from 300 to $12 \mathrm{~K}$ for (c) $\mathrm{HA}_{2} \mathrm{PbI}_{4}$ and (d) $\mathrm{DA}_{2} \mathrm{PbI}_{4}$.

4) over the same temperature range as was used for the TCSPC. In the absorption, we find a slight broadening of the absorption spectra at very low temperatures, but there is no evidence for the formation of a strong additional absorption feature at lower energies for either material. For $\mathrm{HA}_{2} \mathrm{PbI}_{4}$, the main absorption peak gradually red-shifts with reducing temperature. Further, we see the main absorption peak splitting at $175 \mathrm{~K}$, with an energy difference between the peaks on the order of $50 \mathrm{meV}$. No such splitting is observed for $\mathrm{DA}_{2} \mathrm{PbI}_{4}$, for which the absorption peak shows a gradual blue-shift with reducing temperature.

A continuous contraction of the unit cell volume is observed on cooling (Figure 4). In $\mathrm{DA}_{2} \mathrm{PbI}_{4}$ a previously unreported transition to a lower symmetry $P 2_{1} / a$ monoclinic phase is observed at around $200 \mathrm{~K}$ which results in a dramatic change in the lattice parameters. The low-symmetry phase transition involves a change in the stacking of $\mathrm{PbI}_{4}{ }^{2-}$ sheets from eclipsed to staggered. The change in stacking is also associated with puckering of the $\mathrm{PbI}_{4}{ }^{2-}$ sheets out of the $a b$-plane. This symmetry-lowering transition may be the origin of the broadening of the absorption peak in $\mathrm{DA}_{2} \mathrm{PbI}_{4}$ below $200 \mathrm{~K}$. The $\mathrm{PbI}_{4}{ }^{2-}$ plane stretching different amounts in the [100] and [010] directions causes the octahedra to tilt, which can be seen in the ratio of lattice parameters $a$ to $b$, and in the angles between the lead and iodide ions. This can be seen in $\mathrm{DA}_{2} \mathrm{PbI}_{4}$ but not $\mathrm{HA}_{2} \mathrm{PbI}_{4}$ (Supporting Information Figures 7 and 8). Octahedral tilting has been recorded as the cause of blueshifting of bulk 3D organic-inorganic lead halide perovskite in

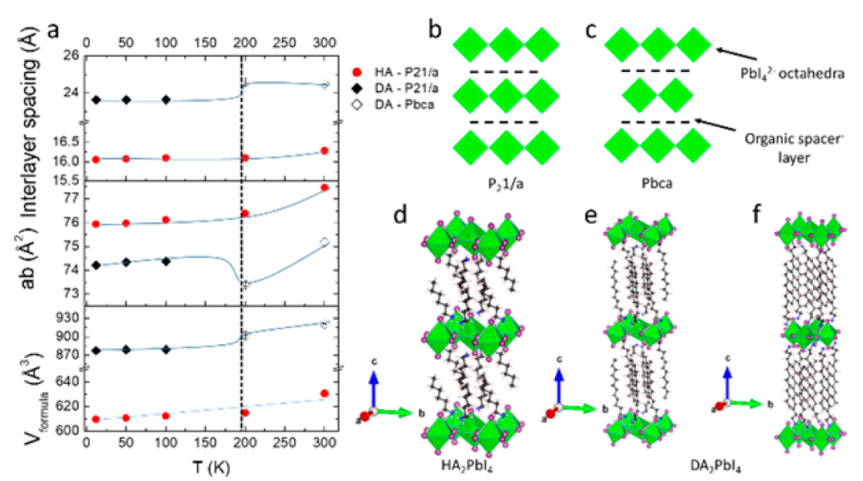

Figure 4. (a) Parameters from Rietveld fits to temperature-dependent powder XRD measurements with lines as guide to the eye. The $y$-axes in figure (a) show the variation in the product of $a$ and $b$ lattice parameters, the interlayer spacing $(c$, or $c / 2$ in the case of the $P b c a$ phase) and formula unit volume $\left(V_{\text {formula }}\right)$. There is a smooth change in the parameters up to $200 \mathrm{~K}$ where there is a phase change in the $\mathrm{DA}_{2} \mathrm{PbI}_{4}$ from monoclinic $P 2_{1} / a$ at low temperature to orthorhombic $P b c a$ above $200 \mathrm{~K}$, while $\mathrm{HA}_{2} \mathrm{PbI}_{4}$ appears to exist as the $P 2_{1} / a$ phase up to room temperature. b) and c) schematic representations of the arrangements of lead iodide octahedra in the $P 2_{1} / a$ and $P b c a$ phases, respectively. (d, e) Structural models for the $P 2_{1} / a$ phases of $\mathrm{HA}_{2} \mathrm{PbI}_{4}$ and $\mathrm{DA}_{2} \mathrm{PbI}_{4}$ respectively. (f) Structural model of the $\mathrm{Pbca}$ phase of $\mathrm{DA}_{2} \mathrm{PbI}_{4} \cdot \mathrm{PbI}_{4}{ }^{2-}$ are shown as green octehedra. Carbon, nitrogen and hydrogen atoms in the aliphatic chains are shown as black, blue and white spheres, respectively.

the past. $^{26}$ This might explain why blue-shifting is seen in $\mathrm{DA}_{2} \mathrm{PbI}_{4}$ but not $\mathrm{HA}_{2} \mathrm{PbI}_{4}$.

Our results exclude the formation of a low-temperature phase common to both materials as the origin of the low-temperature broadband emission, since we do not find evidence for a significant structural transition below room temperature in $\mathrm{HA}_{2} \mathrm{PbI}_{4}$. In order to investigate if the state responsible for redshifted broad emission is accessible at room temperature, but decays nonradiatively on ultrafast time scales compared to the more efficient, higher-energy, emission, we took ultrafast transient PL and transient absorption (TA) measurements (Figure 5). The PL spectra at 1 ps after excitation show one main peak close to the band edge at $520 \mathrm{~nm}\left(\mathrm{HA}_{2} \mathrm{PbI}_{4}\right)$ and
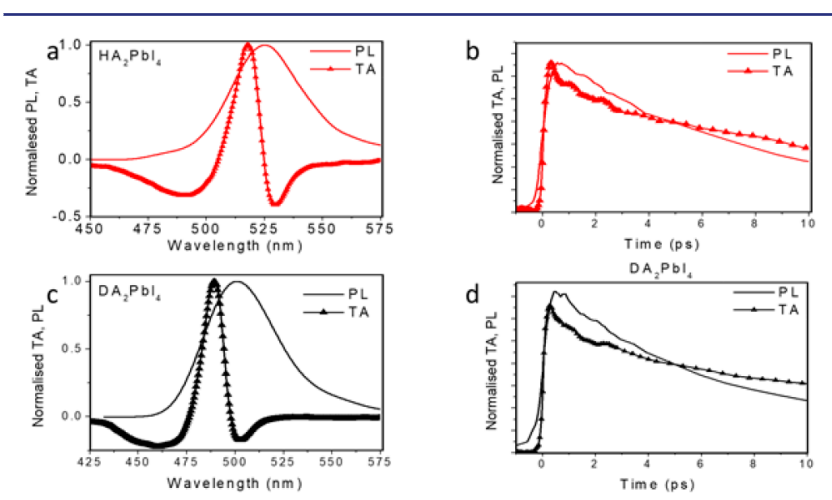

Figure 5. Ultrafast spectroscopy of the investigated $2 \mathrm{D}$ perovskites at room temperature. $\mathrm{PL}$ and TA spectra excited at $400 \mathrm{~nm}$ with a fluence of $11 \mu \mathrm{J} / \mathrm{cm}^{2}$ (TA) and $255 \mu \mathrm{J} / \mathrm{cm}^{2}$ (PL) for $\mathrm{HA}_{2} \mathrm{PbI}_{4}$ and a fluence of $9.7 \mu \mathrm{J} / \mathrm{cm}^{2}$ (TA) and $255 \mu \mathrm{J} / \mathrm{cm}^{2}$ (PL) for $\mathrm{DA}_{2} \mathrm{PbI}_{4}$. TA measurements integrated from 0 to 1 ps for (a) $\mathrm{HA}_{2} \mathrm{PbI}_{4}$ and (b) $\mathrm{DA}_{2} \mathrm{PbI}_{4} \cdot(\mathrm{c}, \mathrm{d})$ Normalized TA and PL kinetics taken at 487-492 nm $\left(\mathrm{DA}_{2} \mathrm{PbI}_{4}\right)$ and $517-521 \mathrm{~nm}\left(\mathrm{HA}_{2} \mathrm{PbI}_{4}\right)$ for the measurements shown in (a) and (b), respectively. 
$495 \mathrm{~nm}\left(\mathrm{DA}_{2} \mathrm{PbI}_{4}\right)$. Although slightly broadened, the PL peaks correspond well with the measured steady-state emission spectra (Figure 1). The TA spectra at 1 ps after excitation show similar features for both materials. A strong and narrow positive feature is present near the band edge due to state filling and bleaching, while a broader negative feature associated with a photoinduced change in refractive index is found at shorter wavelengths. ${ }^{27}$ The TA peak position matches the steady-state absorption peak of both materials. The TA and PL spectra show no distinct features out to $650 \mathrm{~nm}$, the region where one would expect to find a signal from the low-energy state found at low temperatures. The kinetics taken at the peak of PL and TA spectra show slight differences at early times after excitation due to differences in fluence, but have similar decays from 2 ps after excitation, with a time constant of 8 ps. This agrees with a dominant excitonic recombination of the photoexcited states. These results put an upper limit of $100 \mathrm{fs}$ (the time-resolution of our setup) on the lifetime of the lower energy state if it were formed under these conditions.

The coincidence of the buckling of the lead iodide layers with the low-temperature broadband emission is an important indication of a possible (and likely) interplay between the crystalline structure and the measured white emission, as already pointed out in the literature. ${ }^{11-13,15,16}$ However, such coincidence does not provide a strong evidence for a structural origin of this emission and does not rule out other possible causes. Two recent works in fact suggested lattice defects as key players in the white emission, through the formation of selflocalized emissive states. ${ }^{17,28}$ This is also consistent with the extended literature on ionic defects acting as emitting F-centers in inorganic crystals. ${ }^{29-32}$ To investigate this further, we carried out density functional theory calculations of the $\mathrm{HA}_{2} \mathrm{PbI}_{4}$ perovskite, considering both ideal, nondefective crystalline models and defective ones.

Starting from the obtained crystal structure, we first relaxed the atomic positions of the ideal, nondefective $\mathrm{HA}_{2} \mathrm{PbI}_{4}$ layered perovskite at the DFT level (using the PBE functional for the description of the exchange-correlation interaction and neglecting spin-orbit coupling). At this level of theory, the electronic band gap of $2.09 \mathrm{eV}$ is underestimated compared to the experimental value of $2.7 \mathrm{eV} .^{25}$ The PBE error on the band gap can be reduced at a reasonable computational cost by applying a correction within the Hubbard scheme on the iodides $(U=3 \mathrm{eV})$, resulting in a $\mathrm{PBE}+\mathrm{U}$ band gap of $2.71 \mathrm{eV}$ (Figure 6a). In spite of the simplicity of this method we verified that the obtained results are robust, especially in relation to the role of the spin-orbit coupling on the electronic structure of lead halide based perovskite. ${ }^{33}$ Specifically, we checked that the relative energy shifts of the trap states with respect to the semiconductor band edges are almost insensitive to details of the theoretical methodology, with Hubbard corrected PBE $(\mathrm{PBE}+\mathrm{U})$, uncorrected $\mathrm{PBE}$ and spin-orbit coupling corrected $\mathrm{PBE}(\mathrm{PBE}+\mathrm{SOC})$ methods yielding similar results (see Supporting Information Table 1).

DFT investigations suggest that the formation energies for iodide related defects can be very small (down to $0.23 \mathrm{eV}$ under specific growth conditions) in $\mathrm{CH}_{3} \mathrm{NH}_{3} \mathrm{PbI}_{3}$ perovskite. ${ }^{34}$ We repeated these calculations using the same modeling protocol for $\mathrm{HA}_{2} \mathrm{PbI}_{4}$ and found similar results. ${ }^{35,36}$ Our calculations yield a formation energy of $0.67 \mathrm{eV}$ for an iodide interstitial, close to the value reported for the $\mathrm{CH}_{3} \mathrm{NH}_{3} \mathrm{PbI}_{3}$ perovskite ${ }^{37,38}$ (Supporting Information Table 3). In contrast, the computed formation energy of iodide vacancy (Figure 6b) and lead

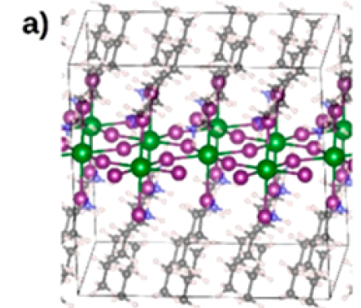

b) ?
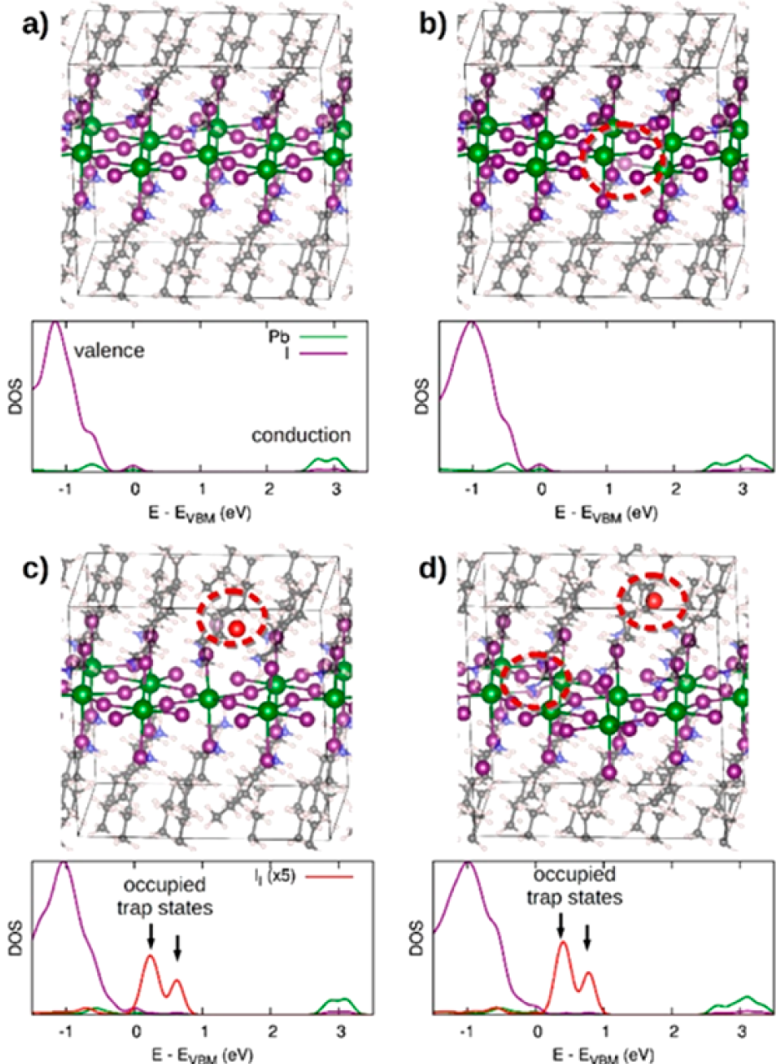

d)
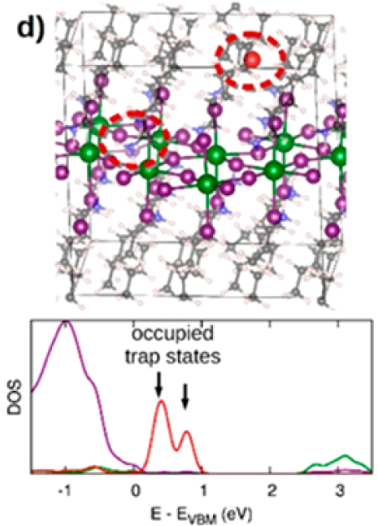

Figure 6. Fully relaxed crystal structures and electronic density of states (DOS) for $\mathrm{HA}_{2} \mathrm{PbI}_{4}$ with (a) nondefective structure; in the presence of iodide ionic defects: (b) iodide vacancy in equatorial position, (c) iodide interstitial $\left(\mathrm{I}_{\mathrm{V}}\right)$ ), and (d) iodide related Frenkel defect. The inorganic network is highlighted, and the defect is indicated by a red circle.

defects is much larger, hence making these other defects extremely unlikely. Finally, the iodide-related Frenkel defect in Figure $6 \mathrm{~d}$ features a formation energy of $1.67 \mathrm{eV}$, which is smaller than the sum of the isolated charged defects, possibly because of overlapping electrostatic and nuclear polarization effects although we cannot exclude purely methodological aspects. ${ }^{35,39}$ Based on these relative formation energies, we thus started our investigations with the likely more numerous iodide point defects (see Figure 6 and Supporting Information Figure 12).

In the frozen lattice of the pristine semiconductor, the removal of one iodide anion in equatorial position with respect to the inorganic sheet forms a trap state partly localized around the defect and located $0.3 \mathrm{eV}$ below the conduction band edge (see Supporting Information Figure 11). Allowing the lattice to relax around this iodide vacancy defect, however, up-shifts the trap state close to the conduction band minimum, resulting in a shallow trap and almost recovering the band gap of the nondefective system (2.62 eV, Figure 6b). Similar "defecttolerance" behavior, that is, the absence of deep trap states upon the formation of ionic defects, has also been reported by Yin et al. for the prototypical $3 \mathrm{D}, \mathrm{CH}_{3} \mathrm{NH}_{3} \mathrm{PbI}_{3}$ perovskite, ${ }^{34}$ and hence appears as a peculiar property of lead-halide perovskite semiconductors. An iodide vacancy in apical position with respect to the inorganic sheet behaves similarly, with almost full recovery of the pristine material electronic structure and a band gap of $2.79 \mathrm{eV}$ (see Supporting Information Figure 
10a). Direct comparison of the energetics of an apical versus equatorial iodide vacancy suggests that the former is favored (considering one defect per four crystal cells, $\sim 4.89 \mathrm{~nm}^{3}$ ) by 14.33-34.18 meV/ $\mathrm{nm}^{3}$ depending on the computational method used (see Supporting Information Table 2).

When an interstitial iodide ion is introduced inside the organic layer as shown in Figure 6c, neither the valence nor conduction band edges nor the DOS show sizable differences with respect to the pristine case. However, three new occupied levels with large contributions around the interstitial iodide ion now appear in the band gap of the material, at $0.65,0.30$, and $0.18 \mathrm{eV}$ above the valence band maximum. Notably, the appearance of deep trap states in the presence of an interstitial iodide is sensitive to the specific iodide position. When the iodide ion is in the middle of the $\mathrm{PbI}_{4}{ }^{2-}$ continuous network (Supporting Information Figure 10b), it strongly distorts the inorganic framework from the regular pattern of the octahedral tilting, typical of both the $3 \mathrm{D}$ and $2 \mathrm{D}$ nondefective case (see Supporting Information Figure 12). As a result, the band gap increases to $\sim 3.1 \mathrm{eV}$ but no trap states are found. When instead the interstitial iodide ion is initially placed between two apical iodides, the final relaxed structure results in a peculiar $\mathrm{Pb}-\mathrm{I}$ packing, where two lead atoms are bound with seven iodides (see Supporting Information Figure 10c). However, the band gap does not show significant difference to the pristine case, with a value of $2.8 \mathrm{eV}$. Finally, we consider the case of the neutral Frenkel-defect associated with the iodine vacancyinterstitial pair, which corresponds to defects formed under stoichiometric conditions, as obviously the creation of an iodide interstitial by ion migration must be accompanied by the formation of a vacancy. The corresponding DOS (Figure 6d) is the combination of the DOS of the two individual defects and the deep occupied trap states induced by the interstitial iodine are still present, at $\sim 0.75,0.45$, and $0.33 \mathrm{eV}$ above the valence band edge.

To sum up, for iodide related defects, the DFT calculations indicate that iodide vacancies do not form intragap states, while iodide interstitials show deep trap states around $0.65 \mathrm{eV}$ above the valence band. The radiative decay of an electron from the perovskite conduction band edge into one of these localized levels is predicted to be centered around $2.1 \mathrm{eV}$, in good agreement with the white emission observed at $2.0 \mathrm{eV}$ (625 $\mathrm{nm})$.

Though these are less likely, we also studied the role of lead vacancies and lead interstitials (Supporting Information Figure 13). As for the iodine case, lead interstitials can form trap states for specific positions of the interstitial within the crystal structure. These unoccupied trap states fall at $\sim 0.79 \mathrm{eV}$ below the conduction band minimum and thus fit reasonably well with the measured energy of the white emission energy. However, the formation energy computed for lead interstitials and vacancies is extremely large, 2.40 and $2.82 \mathrm{eV}$ respectively, hence making these (Supporting Information Table 3) defects thermodynamically unfavorable. This is in line with the situation encountered in the $3 \mathrm{D}$ analogous $\mathrm{CH}_{3} \mathrm{NH}_{3} \mathrm{PbI}_{3}$ perovskite, where a larger formation energy was calculated for lead with respect to iodide related defects. ${ }^{34}$

\section{DISCUSSION}

Our results report the temperature-dependent formation of a long-lived, sub-band-gap state, which gives rise to white light emission in two $\langle 001\rangle$ oriented $2 \mathrm{D}$ perovskite materials, $\mathrm{HA}_{2} \mathrm{PbI}_{4}$ and $\mathrm{DA}_{2} \mathrm{PbI}_{4}$. Rietveld analysis of the XRD measure- ments shows a smooth variation of the unit cell volume from room temperature down to $12 \mathrm{~K}$ with a phase change present in only one of the two $\left(\mathrm{DA}_{2} \mathrm{PbI}_{4}\right)$. This evidence suggests that the observed new emission feature in these materials is not caused by a new phase or crystal structure at low temperature as proposed by Yangui et al. ${ }^{18}$ First-principles calculations attribute the observed low-energy emission to the formation of interstitial iodide related defects acting as color centers. These might be related to the reported formation of color centers in single crystals of $\mathrm{PbI}_{2}$ at wavelengths above $550 \mathrm{~nm}$ under illumination at temperatures above $343 \mathrm{~K}^{40}$ The reported energies and fwhm of the color centers in cyclohexylammonium lead iodide ${ }^{18}$ compare well to the PL signatures we report for the $\mathrm{DA}_{2} \mathrm{PbI}_{4}$ and $\mathrm{HA}_{2} \mathrm{PbI}_{4}$ layered hybrid perovskites investigated.

An important question still remains: why the broad band emission in these $\langle 001\rangle$ oriented $2 \mathrm{D}$ perovskites is observed only at low temperature. In particular, two possibly interconnected defect-related mechanisms can explain the disappearance of the white emission when increasing the temperature: (i) ionic defect migration ${ }^{41-46}$ and (ii) defect recombination. ${ }^{47-49}$ It is now widely established that ions are mobile in 3D hybrid perovskites, hence increase in thermal energy might provide the kinetic energy needed for the defects to diffuse to surface or grain boundaries, where they do not contribute to the optical response of the material. Notably, recent theoretical and experimental observations indicate iodide ions as the most mobile in hybrid materials, which is consistent with the assignment proposed here. ${ }^{46}$ On the other hand, Yamada et al. suggested defect annihilation in $\mathrm{CH}_{3} \mathrm{NH}_{3} \mathrm{PbI}_{3}$ thin films at room temperature, as result of spontaneous, temperature-induced defect healing. ${ }^{47,50}$ Both the diffusion and the annihilation mechanisms are consistent with the temperature dependence observed here as well as the ultrafast spectroscopy at room temperature.

At the same time, it cannot be ruled out that some iodide vacancies and related Frenkel defects are present at room temperature, but recombination of excitations via these states is out-competed by more efficient radiative excitonic recombination events.

The results in this study suggest that white-light emitting LEDs formed from layered 2D lead halide perovskites may be possible by ensuring that the active layer has stable interstitial iodide defects at room temperature. This may be achieved through suitable choice of organic spacer that ensures a crystal structure at room temperature that allows the iodide interstitial to be formed and tunes the dielectric constant in the organic layer to give sufficient charge screening for the defect to be stable at room temperature.

Interesting further investigations would be electron spin resonance performed on these materials to gain insight into the chemical environment of the electrons in the color center, as has previously been performed on lead iodide crystals. ${ }^{51}$ Further, by performing high magnetic field absorption and PL measurements, similar to reports from Hayes and Stoneham, ${ }^{52}$ insights could be gained about the specific spin behavior.

\section{METHODS}

Alkylammonium iodide salts ( $\left.R A \mathrm{I}, R A=\mathrm{C}_{6} \mathrm{H}_{16} \mathrm{~N}, \mathrm{C}_{12} \mathrm{H}_{28} \mathrm{~N}\right)$ were prepared via neutralization of $\mathrm{HI}$ with $R A$. Unreacted species were removed by evaporation. The product was purified by recrystallization in minimal diethyl ether/excess hexane and isolated via vacuum filtration. Films were prepared by spin-coating or drop-casting 
solutions which were prepared by dissolving $R A \mathrm{I}$ and $\mathrm{PbI}_{2}$ powders at a 2.5:1 molar ratio in a 1:0.34 volume ratio mixture of THF and methanol. Films were spin-coated from solutions of $20 \mathrm{mg} / \mathrm{mL}$ at $2000 \mathrm{rpm}$ for $30 \mathrm{~s}$ and annealed for $15 \mathrm{~min}$ at $70 \mathrm{C}^{\circ}$. Films used to prepare powders for XRD were drop-cast from $200 \mathrm{mg} / \mathrm{mL}$ solutions and annealed at $70{ }^{\circ} \mathrm{C}$ for $15 \mathrm{~min}$.

$\mathrm{XRD}$ was performed using a Bruker X-ray D8 Advance diffractometer with $\mathrm{Cu} \mathrm{K} \alpha_{1,2}$ radiation $(\lambda=1.541 \AA)$. Lowtemperature measurements were made on cooling between 300-12 $\mathrm{K}$ using an Oxford Cyrosytem PheniX stage. Spectra were collected with an angular range of $5^{\circ}<2 \theta<60^{\circ}$ and $\Delta \theta=0.01022^{\circ}$ over 60 min. Measurements were made on both as prepared spin coated films and powder samples obtained from the films. The Bruker Topas software ${ }^{22}$ was used to carry out Le Bail ${ }^{53}$ and Rietveld ${ }^{21}$ analysis on film and powder measurements, respectively. Backgrounds were fit with a Chebyshev polynomial function and the peak shape modeled with a pseudo-Voigt function. The positions of the amine molecules were not refined in the Rietveld analysis as the XRD is dominated by scattering from the heavy $\mathrm{PbI}_{4}{ }^{2-}$ sheets.

Steady-state room-temperature absorption spectra were determined by placing spin-coated films on glass in a using an HP 8453 spectrometer. Steady-state RT photoluminescence emission spectra of spin-coated films on glass were measured in an Edinburgh Instruments FLS980 fluorimeter by exciting at with monochromated light with a 2 $\mathrm{nm}$ bandwidth at $480 \mathrm{~nm}$ and spectra were measured with a step size of $1 \mathrm{~nm}$.

Variable angle spectroscopic ellipsometry was measured using a $\mathrm{M}$ 2000 ellipsometer (Woollam Co.) with rotating compensator in the wavelength range from 400 to $800 \mathrm{~nm}$ on samples prepared on synthetic fused silica (Spectrosil) with a native oxide layer. The data were fitted using a layered optical model, based on a film thickness determined by contact profilometry.

PLQE was calculated as per de Mello et al. ${ }^{54}$ by placing semiconductor films in an integrating sphere and were photoexcited using a $405 \mathrm{~nm}$ continuous-wave laser. The laser and the emission signals were measured and quantified using a calibrated Andor iDus DU420A BVF Si detector for the determination of PL quantum efficiency.

Grazing incidence wide-angle X-ray scattering (GIWAXS) measurements on 2D perovskite films were carried out on the I07 beamline at the Diamond Light Source, Didcot, U.K. The X-ray beam energy was $10 \mathrm{keV}$ and the angle of incidence was $0.4^{\circ}$. During measurement each sample was housed inside a custom built sample chamber held under a continuous overpressure of dry He. Data was collected using a Pilatus $2 \mathrm{M}$ detector and analyzed using the DAWN software package (www. dawnsci.org). Silver behenate powder was used as a calibration standard.

Broadband time-integrated absorption spectroscopy (6000i: double beam, Cary) was measured at temperatures between 16 and $300 \mathrm{~K}$. The instrument was calibrated between 300 and $1800 \mathrm{~nm}$. Transmission was measured on Spectrosil) with a He cryostat (CF$\mathrm{V}$, Oxford Instruments) under a $\mathrm{N}_{2}$ atmosphere.

Photoluminescence (PL) kinetics were measured using timecorrelated single photon counting (TC-SPC). Samples prepared on Spectrosil were exposed to a pulsed-UV $(407 \mathrm{~nm}, 1.25 \mathrm{MHz}, 300 \mathrm{ps}$, $360 \mu \mathrm{W}$ ) diode laser (PDL 800-B (trigger), LDH-P-C 400B (laser head), PicoQuant), and the PL was recorded after external triggering. Measurements at temperatures between 50 and $300 \mathrm{~K}$ were conducted using a cryostat (CF-V, Oxford Instruments).

A commercially available Ti:sapphire amplifier (Spectra-Physics Solstice Ace) operating at $1 \mathrm{kHz}$ generated $100 \mathrm{fs}$ pulses centered around $800 \mathrm{~nm}$. The laser output was then split to generate both the pump and probe pulses. For the pump pulse, the output of the Ti:sapphire amplifier was frequency doubled in a $1 \mathrm{~mm}$ thick BBO crystal (Eksma Optics) to give a narrowband $400 \mathrm{~nm}$ pump with an approximately $100 \mathrm{fs}$ duration. The intensity of these pump pulses was controlled using a variable neutral density filter (Thorlabs). For the broadband probe, a small portion of the $800 \mathrm{~nm}$ beam was focused into a $5 \mathrm{~mm}$ calcium fluoride crystal (Thorlabs) to generate a white light supercontinuum. The crystal was translated vertically at $5 \mathrm{~Hz}$ on a home-built stage to avoid irreversible pump-induced damage. The transmitted probe pulses were then collected with a silicon dual-line array detector (Hamamatsu S8381-1024Q) driven and read out by a custom-built board from Stresing Entwicklungsbüro.

A Ti:sapphire amplifier system (Spectra-Physics Solstice) operating at $1 \mathrm{kHz}$ generated $80 \mathrm{fs}$ pulses that were split into the pump and probe beam arms. The pump beam was generated by second harmonic generation (SHG) in a BBO crystal and focused onto the sample. Photoluminescence is collimated using a silver off-axis parabolic mirror and focused onto the gate medium. About $80 \mu \mathrm{J} /$ pulse of the $800 \mathrm{~nm}$ laser output is used for the gate beams, which is first raised $25 \mathrm{~mm}$ above the plane of the PL to produce a boxcar geometry and split into a pair of gate beams using a 50/50 beam splitter. The gate beams are focused onto the gate medium (fused silica), crossing at an angle of $\sim 5^{\circ}$ and overlapping with the focused PL. The two gate beams interfere and create a transient grating in the gate medium due to a modulation of the refractive index via the optical Kerr effect. ${ }^{55}$ Temporal overlap between the two gate beams is achieved via a manual delay stage. The PL is then deflected on the transient grating causing a spatial separation of the gated signal from the PL background. Two lenses collimate and focus the gated signal onto the spectrometer entrance (Princeton Instruments SP 2150) after long- and short-pass filters remove scattered pump and gate light, respectively. Gated PL spectra are measured using an intensified CCD camera (Princeton Instruments, PIMAX4). The $(\sim 10 \mathrm{~ns})$ electronic shutter of the intensified CCD camera was used to further suppress long-lived PL background. PL spectra at each gate time delay are acquired from $\sim 10000$ laser shots. The time delay between pump and gate beams is controlled via a motorized optical delay line on the excitation beam path and a LabVIEW data acquisition program. ${ }^{55}$

Electronic structure calculations and cell relaxation were performed within the planewave-pseudopotential approach, as implemented in the PWSCF program of the Quantum-Espresso suite. ${ }^{56}$ For the expansion of the wave function and of the DOS, a plane wave kinetic energy cutoff of 25 and $200 \mathrm{Ry}$, respectively, was used along with ultrasoft. ${ }^{57}$ scalar relativistic pseudopotentials and the PBE functional for the electronic exchange-correlation. ${ }^{58}$ The electronic structure was calculated in the $\Gamma$ point of the first Brillouin zone. The Grimme correction scheme was used to take into account the van der Waals interactions, which are of particular importance for the interaction among the alkyl-chains. ${ }^{59}$ The present computational setup is similar to that used for similar models for case of $3 \mathrm{D}$ perovskites. ${ }^{50}$

\section{ASSOCIATED CONTENT}

\section{S Supporting Information}

The Supporting Information is available free of charge on the ACS Publications website at DOI: 10.1021 /jacs.7b10223.

Crystal data for dodecylammonium lead iodide $\mathrm{DA}_{2} \mathrm{PbI}_{4}$ (CIF)

Optical spectra, crystallographic data and analysis, and further details of the computational results and techniques (PDF)

The data underlying this paper are available at https:// doi.org/10.17863/CAM.15742.

\section{AUTHOR INFORMATION}

\section{Corresponding Authors}

*epb24@cam.ac.uk

*fd297@cam.ac.uk

ORCID ${ }^{\circ}$

Edward P. Booker: 0000-0001-8155-8206

Claudio Quarti: 0000-0002-5488-1216

Neil C. Greenham:0000-0002-2155-2432

Notes

The authors declare no competing financial interest. 


\section{ACKNOWLEDGMENTS}

The work was supported by The Centre of Doctoral Training in New and Sustainable Photovoltaics Grant EP/L01551X/1 and the NanoDTC ESPSRC Grant EP/L015978/1. T.H.T. thanks an EPSRC iCASE studentship for funding. J.M.R. thanks the Winton Program for Sustainability and the Cambridge Trust. N.J.L.K.D. thanks the Ernest Oppenhimer Trust for a research fellowship. F.D. thanks the Winton Program for Sustainability for a research fellowship. The work was supported by the Interuniversity Attraction Pole program of the Belgian Federal Science Policy Office (PAI 6/27) and FNRS-F.R.S. Computational resources have been provided by the Consortium des Équipements de Calcul Intensif (CÉCI), funded by the Fonds de la Recherche Scientifique de Belgique (F.R.S.-FNRS) under Grant No. 2.5020.11. D.B. is a FNRS Research Director. We thank the Diamond Light Source for GIWAXS beamtime access through session SI14886-1.

\section{REFERENCES}

(1) Green, M. A.; Emery, K.; Hishikawa, Y.; Warta, W.; Dunlop, E. D. Prog. Photovoltaics 2016, 24, 905-913.

(2) Saliba, M.; Matsui, T.; Seo, J.-Y.; Domanski, K.; Correa-Baena, J.P.; Nazeeruddin, M. K.; Zakeeruddin, S. M.; Tress, W.; Abate, A.; Hagfeldt, A.; Grätzel, M. Energy Environ. Sci. 2016, 9, 1989-1997.

(3) Zhang, S.; Yi, C.; Wang, N.; Sun, Y.; Zou, W.; Wei, Y.; Cao, Y.; Miao, Y.; Li, R.; Yin, Y.; Zhao, N.; Wang, J.; Huang, W. Adv. Mater. 2017, 29, 1606600.

(4) You, J.; Meng, L.; Song, T.-B.; Guo, T.-F.; Yang, Y.; Chang, W.H.; Hong, Z.; Chen, H.; Zhou, H.; Chen, Q.; Liu, Y.; De Marco, N.; Yang, Y. Nat. Nanotechnol. 2015, 11, 75-81.

(5) Bryant, D.; Aristidou, N.; Pont, S.; Sanchez-Molina, I.; Chotchunangatchaval, T.; Wheeler, S.; Durrant, J. R.; Haque, S. A. Energy Environ. Sci. 2016, 9, 1655-1660.

(6) Niu, G.; Guo, X.; Wang, L. J. Mater. Chem. A 2015, 3, 89708980.

(7) Quan, L. N.; Yuan, M.; Comin, R.; Voznyy, O.; Beauregard, E. M.; Hoogland, S.; Buin, A.; Kirmani, A. R.; Zhao, K.; Amassian, A.; Kim, D. H.; Sargent, E. H. J. Am. Chem. Soc. 2016, 138, 2649-2655.

(8) Stoumpos, C. C.; Cao, D. H.; Clark, D. J.; Young, J.; Rondinelli, J. M.; Jang, J. I.; Hupp, J. T.; Kanatzidis, M. G. Chem. Mater. 2016, 28, 2852-2867.

(9) Cao, D. H.; Stoumpos, C. C.; Farha, O. K.; Hupp, J. T.; Kanatzidis, M. G. J. Am. Chem. Soc. 2015, 137, 7843-7850.

(10) Blancon, J.-C.; Tsai, H.; Nie, W.; Stoumpos, C. C.; Pedesseau, L.; Katan, C.; Kepenekian, M.; Soe, C. M. M.; Appavoo, K.; Sfeir, M. Y.; et al. Science 2017, 355, 1288-1292.

(11) Cortecchia, D.; Neutzner, S.; Srimath Kandada, A. R.; Mosconi, E.; Meggiolaro, D.; De Angelis, F.; Soci, C.; Petrozza, A. J. Am. Chem. Soc. 2017, 139, 39-42.

(12) Cortecchia, D.; Yin, J.; Bruno, A.; Lo, S.-Z. A.; Gurzadyan, G. G.; Mhaisalkar, S.; Brédas, J.-L.; Soci, C. J. Mater. Chem. C 2017, 5, 2771-2780.

(13) Dohner, E. R.; Jaffe, A.; Bradshaw, L. R; Karunadasa, H. I. J. Am. Chem. Soc. 2014, 136, 13154-13157.

(14) Cheng, Z.; Lin, J. CrystEngComm 2010, 12, 2646-2662.

(15) Mao, L.; Wu, Y.; Stoumpos, C. C.; Wasielewski, M. R.; Kanatzidis, M. G. J. Am. Chem. Soc. 2017, 139, 5210-5215.

(16) Dohner, E. R.; Hoke, E. T.; Karunadasa, H. I. J. Am. Chem. Soc. 2014, 136, 1718-1721.

(17) Hu, T.; Smith, M. D.; Dohner, E. R.; Sher, M.-J.; Wu, X.; Trinh, M. T.; Fisher, A.; Corbett, J.; Zhu, X.-Y.; Karunadasa, H. I.; Lindenberg, A. M. J. Phys. Chem. Lett. 2016, 7, 2258-2263.

(18) Yangui, A.; Pillet, S.; Mlayah, A.; Lusson, A.; Bouchez, G.; Triki, S.; Abid, Y.; Boukheddaden, K. J. Chem. Phys. 2015, 143, 224201.

(19) Billing, D. G.; Lemmerer, A. Acta Crystallogr., Sect. B: Struct. Sci. 2007, 63, 735-747.
(20) Billing, D. G.; Lemmerer, A. New J. Chem. 2008, 32, 17361746.

(21) Rietveld, H. M. J. Appl. Crystallogr. 1969, 2, 65-71.

(22) Coelho, A. A.; Evans, J.; Evans, I.; Kern, A.; Parsons, S. Powder Diffr. 2011, 26, S22-S25.

(23) Ishihara, T.; Takahashi, J.; Goto, T. Phys. Rev. B: Condens. Matter Mater. Phys. 1990, 42, 11099-11107.

(24) Ishihara, T.; Takahashi, J.; Goto, T. Solid State Commun. 1989, 69, 933-936.

(25) Tanaka, K.; Sano, F.; Takahashi, T.; Kondo, T.; Ito, R.; Ema, K. Solid State Commun. 2002, 122, 249-252.

(26) Amat, A.; Mosconi, E.; Ronca, E.; Quarti, C.; Umari, P.; Nazeeruddin, M. K.; Grätzel, M.; De Angelis, F. Nano Lett. 2014, 14, 3608-3616.

(27) Price, M. B.; Butkus, J.; Jellicoe, T. C.; Sadhanala, A.; Briane, A.; Halpert, J. E.; Broch, K.; Hodgkiss, J. M.; Friend, R. H.; Deschler, F. Nat. Commun. 2015, 6, 8420.

(28) Cortecchia, D.; Yin, J.; Bruno, A.; Lo, S.-Z. A.; Gurzadyan, G. G.; Mhaisalkar, S.; Brédas, J.-L.; Soci, C. J. Mater. Chem. C 2017, 5, $2771-2780$

(29) Seitz, F. Rev. Mod. Phys. 1954, 26, 7-94.

(30) Kanzig, W. J. Phys. Chem. Solids 1960, 17, 80-87.

(31) Kao, C.; Rowan, L. G.; Slifkin, L. M. Phys. Rev. B: Condens. Matter Mater. Phys. 1990, 42, 3142-3151.

(32) Rinke, P.; Schleife, A.; Kioupakis, E.; Janotti, A.; Rödl, C.; Bechstedt, F.; Scheffler, M.; Van De Walle, C. G. Phys. Rev. Lett. 2012, 108, 126404.

(33) Even, J.; Pedesseau, L.; Jancu, J. M.; Katan, C. J. Phys. Chem. Lett. 2013, 4, 2999-3005.

(34) Yin, W.-J.; Shi, T.; Yan, Y. Appl. Phys. Lett. 2014, 104, 063903.

(35) Lany, S.; Zunger, A. Phys. Rev. B - Condens. Matter Mater. Phys. 2008, 78, 17-20.

(36) Freysoldt, C.; Grabowski, B.; Hickel, T.; Neugebauer, J.; Kresse, G.; Janotti, A.; Van De Walle, C. G. Rev. Mod. Phys. 2014, 86, $253-$ 305.

(37) Yin, W.; Shi, T.; Yan, Y.; Yin, W.; Shi, T.; Yan, Y. Appl. Phys. Lett. 2014, 104, 063903.

(38) Agiorgousis, M. L.; Sun, Y. Y.; Zeng, H.; Zhang, S. J. Am. Chem. Soc. 2014, 136, 14570-14575.

(39) Freysoldt, C.; Neugebauer, J.; Van De Walle, C. G. Phys. Rev. Lett. 2009, 102, 1-4.

(40) Forty, A. J. Br. J. Appl. Phys. 1963, 14, 3-9.

(41) Unger, E. L.; Hoke, E. T.; Bailie, C. D.; Nguyen, W. H.; Bowring, A. R.; Heumüller, T.; Christoforo, M. G.; McGehee, M. D. Energy Environ. Sci. 2014, 7, 3690-3698.

(42) Xiao, Z.; Yuan, Y.; Shao, Y.; Wang, Q.; Dong, Q.; Bi, C.; Sharma, P.; Gruverman, A.; Huang, J. Nat. Mater. 2014, 14, 193-198.

(43) Azpiroz, J. M.; Mosconi, E.; Bisquert, J.; De Angelis, F. Energy Environ. Sci. 2015, 8, 2118-2127.

(44) Richardson, G.; O’Kane, S. E. J.; Niemann, R. G.; Peltola, T. A.; Foster, J. M.; Cameron, P. J.; Walker, A. B. Energy Environ. Sci. 2016, 9, 1476-1485.

(45) Eames, C.; Frost, J. M.; Barnes, P. R. F.; O’Regan, B. C.; Walsh, A.; Islam, M. S. Nat. Commun. 2015, 6, 7497.

(46) Minns, J. L.; Zajdel, P.; Chernyshov, D.; van Beek, W.; Green, M. A. Nat. Commun. 2017, 8, 15152.

(47) Yamada, Y.; Endo, M.; Wakamiya, A.; Kanemitsu, Y. J. Phys. Chem. Lett. 2015, 6, 482-486.

(48) Mosconi, E.; Meggiolaro, D.; Snaith, H. J.; Stranks, S. D.; De Angelis, F. Energy Environ. Sci. 2016, 9, 3180-3187.

(49) Ghosh, S.; Pal, S. K.; Karki, K. J.; Pullerits, T. ACS Energy Lett. 2017, 2, 2133-2139.

(50) Quarti, C.; Mosconi, E.; De Angelis, F. Chem. Mater. 2014, 26, 6557-6569.

(51) Patankar, A. V.; Schneider, E. E. J. Phys. Chem. Solids 1966, 27, $575-579$.

(52) Hayes, W.; Stoneham, A. M. Defects and Defect Processes in Nonmetallic Solids; Wiley, 1985. 
(53) Le Bail, A.; Duroy, H.; Fourquet, J. L. Mater. Res. Bull. 1988, 23, 447-452.

(54) de Mello, J. C.; Wittmann, H. F.; Friend, R. H. Adv. Mater. 1997, 9, 230-232.

(55) Chen, K.; Gallaher, J. K.; Barker, A. J.; Hodgkiss, J. M. J. Phys. Chem. Lett. 2014, 5, 1732-1737.

(56) Giannozzi, P.; Baroni, S.; Bonini, N.; Calandra, M.; Car, R.; Cavazzoni, C.; Ceresoli, D.; Chiarotti, G. L.; Cococcioni, m.; Dabo, I.; Dal Corso, A.; Fabris, S.; Fratesi, G.; de Gironcoli, S.; Gebauer, R.; Gerstmann, U.; Gougoussis, C.; Kokalj, A.; Lazzeri, M.; Martin-Samos, L.; Marzari, N.; Mauri, F.; Mazzarello, R.; Paolini, S.; Pasquarello, A.; Paulatto, L.; Sbraccia, C.; Scandolo, S.; Sclauzero, G.; Seitsonen, A. P.; Smogunov, A.; Umari, P.; Wentzcovitch, R. M. J. Phys.: Condens. Matter 2009, 21, 395502.

(57) Vanderbilt, D. Phys. Rev. B: Condens. Matter Mater. Phys. 1990, 41, 7892-7895.

(58) Perdew, J. P.; Burke, K.; Ernzerhof, M. Phys. Rev. Lett. 1996, 77, 3865-3868.

(59) Truhlar, D. G. J. Comput. Chem. 2009, 28, 1787-1799. 